Aim of the study: Despite widespread use of pharmacological prophylaxis, venous thromboembolism (VTE) still constitutes a common complication in cancer patients. The aim of the study was to analyse the safety of low-molecular-weight heparins (LMWH) in the prevention of VTE in surgically-treated cancer patients.

Material and methods: A total of 5207 cancer patients $(44.5 \%$ men and $55.5 \%$ women) aged $16-97$ years participated in a prospective observational study conducted in 13 Polish cancer centres in 2005-2008. This cohort in cluded 4782 subjects who were treated surgically and received LMWH as a pharmacological prophylaxis for VTE prior to or after the surgery. The incidence of haemorrhagic complications and thrombocytopaenia was analysed in this cohort, along with intra-hospital mortality.

Results: Mean duration of LMWH administration was $9.4 \pm 7.8$ days. Haemorrhagic complications: heavy $(n=15)$ or light bleeding $(n=299)$, were observed in 314 patients (6.5\%). A total of 314 patients $(6.5 \%)$ presented with haemorrhagic complications: heavy $(n=15,0.3 \%)$ or light bleeding $(n=299$, $6.3 \%)$. Four cases of heavy bleeding. gastrointestinal bleeding $(n=2)$, retroperitoneal bleeding $(n=1)$, and central nervous system bleeding $(n=1)$, were classified as definitely related to LMWH. No significant association was found between the incidence of haemorrhagic complications and the type of administered LWMH ( $p=0.523)$. No cases of thrombocytopaenia or deaths related to administration of $\mathrm{LMWH}$ were reported.

Conclusions: LMWH seems to be a safe form of pharmacological prophylaxis for VTE in surgically-treated cancer patients.

Key words: venous thromboembolism, pharmacological prophylaxis, low-molecular-weight heparins.

Contemp Oncol (Pozn) 2017; 21 (2): 152-156 DOI: https://doi.org/10.5114/wo.2017.68624

\section{The safety of low-molecular- weight heparins in the prevention of venous thromboembolism in surgically-treated cancer patients: results of a multicentre observational study}

\author{
Tomasz Olesiński ${ }^{1}$, Anna Fijałkowska², Andrzej Rutkowski ${ }^{1}$
}

1Department of Oncological Gastroenterology, Maria Sklodowska-Curie Memorial Cancer Center and Institute of Oncology, Warsaw, Poland

${ }^{2}$ Department of Cardiology, Mother and Child Institute, Warsaw, Poland

\section{Introduction}

Low-molecular-weight heparins (LMWH) are still a recommended therapy in the prevention and treatment of venous thromboembolism (VTE) [1]. Despite this, Eurostat data imply that mortality due to thromboembolic complications in the EU states is still greater than the cumulative mortality due to AIDS, breast cancer, and road accidents [1]

Cancer patients constitute a particular risk group for thromboembolic complications. This is inter alia associated with the asymptomatic course of VTE $[1,2]$. Pulmonary embolism (PE), still diagnosed no earlier than on autopsy in $70 \%$ of cases, constitutes the principal direct course of VTE-related mortality [2-4]. Importantly, up to $93 \%$ of patients who die due to PE do not receive appropriate prevention/therapy of VTE, which is a leading preventable cause of death in this condition [5].

Owing a widespread use of $\mathrm{LMWH}$, an array of adverse events related to these agents have been identified, which raised concerns about their safety, especially in cancer patients. However, analysis of available evidence in this matter should be adjusted for heterogeneous characteristics of LMWH-administered patients, the setting and duration of their use, therapeutic protocols and dosage regimens, concomitant therapies, comorbidities, and treatment objectives. Therapeutic guidelines, especially regarding long-term administration of $\mathrm{LMWH}$, should also consider the preferences and situation of a given patient.

The aim of this multicentre observational study was to analyse the safety of LMWH in the prevention of VTE in surgically-treated cancer patients.

\section{Material and methods}

Between January 2005 and December 2008, a prospective observational multicentre study "ONCO - Venous Thromboembolism Prevention in Cancer Patients" (protocol no. XRP4563C_5041) was conducted in 13 Polish cancer centres (Table 1). Inclusion and exclusion criteria of the study are listed in Table 2.

The study included a total of 5207 cancer patients: 2317 (44.5\%) men aged 17-92 years (median 63 years) and 2890 (55.5\%) women aged 16-97 years (median 61 years). A detailed age structure of the study subjects is presented in Table 3. A total of 5051 (97\%) patients were treated surgically; additionally, 634 (12.2\%) hospitalised patients received radiotherapy and 
Table 1. List of cancer centres participating in the study

\begin{tabular}{|c|c|}
\hline Institution & Organisational unit \\
\hline Lower Silesian Regional Comprehensive Cancer Centre in Wroclaw & 1st Department of Oncological Surgery \\
\hline Centre of Oncology, Maria Sklodowska-Curie Memorial Institute, Krakow Branch & Clinic of Oncological Surgery \\
\hline Centre of Oncology, Maria Sklodowska-Curie Memorial Institute, Krakow Branch & Department of Oncological Surgery \\
\hline Nicolaus Copernicus Provincial Specialist Hospital in Lodz & Clinic of Oncological Surgery \\
\hline Nicolaus Copernicus Provincial Specialist Hospital in Lodz & Department of Oncological Surgery \\
\hline Regional Comprehensive Cancer Centre in Bydgoszcz & Clinical Department of Oncological Surgery \\
\hline Provincial Specialist Hospital in Slupsk & Department of Oncological Surgery and Breast Diseases \\
\hline Provincial Integrated Hospital in Elblag & Department of Oncological Surgery \\
\hline Centre of Oncology, Maria Sklodowska-Curie Memorial Institute in Warsaw & Clinic of Upper Gastrointestinal Malignancies \\
\hline Bialystok Regional Comprehensive Cancer Centre & Department of Oncological Surgery \\
\hline Independent Public Clinical Hospital No. 1, Medical University of Lublin & Clinic of Oncological Surgery \\
\hline Cancer Centre in Brzozow & Department of Oncological Surgery \\
\hline Centre of Oncology, Maria Sklodowska-Curie Memorial Institute, Gliwice Branch & Clinic of Oncological Surgery \\
\hline
\end{tabular}

Table 2. Inclusion and exclusion criteria of the study

$\begin{array}{ll}\text { Inclusion criteria } & \begin{array}{l}\text { Age } \geq 18 \text { years } \\ \text { Diagnosis of cancer }\end{array} \\ \text { Exclusion criteria } & \begin{array}{l}\text { Participation in another clinical trial } \\ \text { Hospitalisation due to a newly diagnosed VTE requiring implementation of anticoagulation therapy } \\ \text { Long-term anticoagulation therapy due to atrial fibrillation, implantation of prosthetic cardiac valves, acute } \\ \text { coronary syndrome, or VTE }\end{array} \\ \text { VTE - venous thromboembolism }\end{array}$

Table 3. Sex and age structure of the study subjects

$\begin{array}{cccc}\text { Age (years) } & \text { Total }(n=5207) & \text { Women }(n=2890) & \text { Men }(n=2317) \\ \leq 40 & 289(6 \%) & 166(6 \%) & 123(5 \%) \\ 41-60 & 2042(39 \%) & 1235(43 \%) & 807(35 \%) \\ 61-80 & 2615(50 \%) & 1332(46 \%) & 1283(56 \%) \\ >80 & 261(5 \%) & 157(5 \%) & 104(4 \%)\end{array}$

380 (7.3\%) chemotherapy. Mean duration of hospital stay was $12.7 \pm 4.2$ days.

The risk of VTE in the study subjects was estimated, based on modified Kucher scores (Appendix 1), as very high ( $\geq 5$ points; $n=3733,71.7 \%$ ), high (3-4 points; $n=1182$, $22.7 \%$ ), moderate (2 points; $n=125,2.4 \%$ ), or low (0-1 points; $n=167,3.2 \%$ ). Pharmacological prophylaxis of VTE with $\mathrm{LMWH}$, lasting for $9.4 \pm 7.8$ days on average, was implemented in 4782 (91.8\%) patients, starting on admission to hospital ( $n=407,8.5 \%), 12 \mathrm{~h}(n=3552,74.3 \%)$ or $2 \mathrm{~h}$ prior to the surgery $(n=275,5.8 \%), 12 \mathrm{~h}(n=323,6,8 \%)$ or 24 h post-surgery $(n=218,4.6 \%)$.

Despite implementations of the LMWH prophylaxis, the signs suggestive of deep vein thrombosis and PE were observed in 18 (0.37\%) and 19 (0.39\%) patients, respectively. Therefore, secondary prevention was implemented in 21 (0.4\%), LMWH in 18, and vitamin $\mathrm{K}$ antagonist in three study subjects. After discharge from hospital, 2255 (47\%) of the patients were subjected to a physiotherapy prevention of VTE for an average period of $20 \pm 8.2$ days.

The incidence of haemorrhagic complications reported after administration of LMWH was analysed in 4782 surgical-
Table 4. Bleeding severity criteria

$\begin{array}{ll}\text { Light bleeding } & \text { Heavy bleeding } \\ \text { Microscopic haematuria } & \text { Gastrointestinal bleeding } \\ \text { Gingival bleeding } & \text { Intracranial bleeding } \\ \text { Epistaxis } & \text { Intra-spinal bleeding } \\ \text { Wound haematoma } & \text { Intraocular bleeding } \\ \text { Injection site haematoma } & \text { Retroperitoneal bleeding } \\ & \text { Intra-articular bleeding } \\ & \text { Gross haematuria }\end{array}$

ly-treated cancer patients. The complications were classified as heavy or light bleeding, in line with the criteria presented in Table 4, and according to their drug-relatedness (not related, unlikely to be related, or definitely related to LMWH).

The results are presented as basic statistics: arithmetic means, standard deviations, medians, and ranges for continuous variables and distributions (along with 95\% confidence intervals, $95 \% \mathrm{Cl}$ ) for discrete variables. Distributions of discrete variables were compared with Fisher exact test. All statistical calculations were conducted, and the threshold of statistical significance for all analyses was defined at $p \leq 0.05$. 
Table 5. Distribution of the study subjects according to LMWH dose

\begin{tabular}{|c|c|c|c|c|c|c|c|}
\hline \multirow[t]{2}{*}{ LMWH } & \multirow[t]{2}{*}{ Total } & \multicolumn{3}{|c|}{ Lower dose } & \multicolumn{3}{|c|}{ Larger dose } \\
\hline & & dose & $n$ & $\%$ & dose & $n$ & $\%$ \\
\hline Enoxaparin & 4219 & $20 \mathrm{mg}$ & 1042 & 24.7 & $40 \mathrm{mg}$ & 3177 & 75.3 \\
\hline Nadroparin & 481 & $0.3 \mathrm{ml}$ & 313 & 65.0 & $0.6 \mathrm{ml}$ & 168 & 35.0 \\
\hline Dalteparin & 53 & - & - & & $0.2 \mathrm{ml}$ & 53 & 100.0 \\
\hline Parnaparin & 29 & $0.3 \mathrm{ml}$ & 24 & 82.0 & $0.4-0.6 \mathrm{ml}$ & 5 & 18.0 \\
\hline Total & 4782 & - & 1379 & 28.0 & - & 3403 & 72.0 \\
\hline
\end{tabular}

LMWH - low-molecular-weight heparins

Table 6. Incidence of haemorrhagic complications overall and stratified according to LMWH type

\begin{tabular}{|c|c|c|c|c|c|c|}
\hline \multirow[t]{2}{*}{ LMWH } & \multicolumn{2}{|c|}{ Haemorrhagic complications overall } & \multicolumn{2}{|c|}{ Light bleeding } & \multicolumn{2}{|c|}{ Heavy bleeding } \\
\hline & $n$ & $\%(95 \% \mathrm{Cl})$ & $n$ & $\%$ & $n$ & $\%$ \\
\hline Enoxaparin & 273 & $6.5(5.76-7.28)$ & 259 & 94.9 & 14 & 5.1 \\
\hline Nadroparin & 23 & $4.8(3.09-7.18)$ & 22 & 95.7 & 1 & 4.3 \\
\hline Dalteparin & 16 & 29.6 (17.98-43.61) & 16 & 100.0 & 0 & 0.0 \\
\hline Parnaparin & 2 & $7.1(0.88-23.5)$ & 2 & 100.0 & 0 & 0.0 \\
\hline Total & 314 & $6.5(5.8-7.3)$ & 299 & 95.2 & 15 & 4.8 \\
\hline
\end{tabular}

LMWH-low-molecular-weight heparins

Table 7. Changes in the therapeutic approach implemented in patients with haemorrhagic complications

$\begin{array}{lcc}\text { Change in the therapeutic approach } & n & \% \\ \text { Discontinuation of LMWH } & 18 & 5.7 \\ \text { Blood transfusion } & 18 & 5.7 \\ \text { Prolongation of hospital stay } & 10 & 3.2 \\ \text { Additional diagnostic tests } & 2 & 0.6 \\ \text { Surgical intervention } & 6 & 1.9 \\ \text { Pharmacotherapy } & 7 & 2.0 \\ \text { Change of VTE prophylaxis } & 3 & 0.9 \\ \text { Hospitalisation at intensive care unit } & 1 & 0.3 \\ \text { None } & 249 & 79.3\end{array}$

LMWH - low-molecular-weight heparins; VTE - venous thromboembolism

\section{Results}

Most patients ( $n=3457,72.3 \%$ ) received larger doses of LMWH (Table 5). Aside from LMWH, also other agents with a potential influence on the coagulation system (nonsteroidal anti-inflammatory drugs, dextran, acetylsalicylic acid) were given postoperatively in 1770 (37\%) patients.

Haemorrhagic complications were observed in 314 patients $(6.5 \%, 95 \% \mathrm{Cl}: 5.8-7.3 \%)$. Heavy bleeding was reported in 15 cases $(0.3 \%, 95 \% \mathrm{Cl}: 0.2-0.5 \%)$ and light bleeding in 299 (6.3\%, 95\% Cl: 5.6-7.0\%). Among 15 cases of heavy bleeding, there were three eventually classified as not related to LMWH, eight unlikely to be related, and four definitely related. Haemorrhagic complications classified as definitely related to LMWH included gastrointestinal bleeding $(n=2)$, retroperitoneal bleeding $(n=1)$, and central nervous system bleeding $(n=1)$. No significant association was found between the incidence of haemorrhagic complications and the type of administered LWMH ( $p=0.523$, Table 6).
The subset of patients with heavy or light bleeding included 65 individuals who required a change in the therapeutic approach: discontinuation of pharmacological prophylaxis for VTE $(n=18)$, blood transfusion $(n=18)$, prolongation of hospital stay $(n=10)$, or other intervention (Table 7).

No cases of thrombocytopaenia were reported in $L M$ $\mathrm{WH}$-administered patients. A total of 20 patients $(0.4 \%$, 95\% Cl: $0.3-0.6 \%)$ died during hospital stay due to progression of cancer $(n=7)$, heart failure $(n=6)$, cardiac arrest $(n=3)$, acute myocardial infarction $(n=2), \operatorname{PE}(n=1)$, and hypertensive chronic kidney disease $(n=1)$. None of these deaths were related to LMWH administration.

\section{Discussion}

Surgical treatment of cancer patients is associated with high risk of thromboembolic complications, usually being a consequence of the extensive procedure (regional resection involving both the affected organ and its lymphatic drainage area) and cancer-induced overactivity of the coagulation system $[6,7]$. As a result, mortality risk due to VTE in surgically-treated cancer patients is at least twice as high as in the general population [8], and thromboembolic complications are considered the second (after tumour progression) cause of death in this group $[9,10]$. The pathomechanism of this phenomenon is complex. Cancer cells may interact with the coagulation system directly through secretion of tissue factor (TF) or indirectly, due to release of pro-inflammatory cytokines [6]. Venous thromboembolism may be also a consequence of endothelial injury, or activation of leukocytes and thrombocytes [6]. Furthermore, the presence of malignancy does not exclude the involvement of other risk factors for VTE typical for the general population $[6,7]$. 
The increased risk of VTE in cancer patients was reflected in the therapeutic guidelines of the American College of Chest Physicians (ACCP) [11] and the recommendations of Polish experts [12]. Due to increased risk of VTE, cancer patients should receive higher doses of LMWH for at least four weeks within the framework of primary prophylaxis, and for 3-6 months (grade 1A recommendation) or until the end of anticancer treatment (grade $1 \mathrm{C}$ recommendation) in secondary prevention [11, 12]. Importantly, treatment of thromboembolic episodes in cancer patients was shown to be costlier than covering this population with the primary prevention [8].

Although administration of $\mathrm{LMWH}$ as a form of primary prevention in cancer patients receiving chemotherapy in an inpatient setting results in a $67 \%$ decrease in the risk of VTE, the risk of this complication should always be assessed on an individual basis [8]. However, such approach is not a routine component of the ACCP guidelines [11]. Optimisation of the therapy (selection of a therapeutic agent and dosage) to minimise the risk of haemorrhagic complications is still problematic. The situation is additionally complicated by the fact that cancer patients usually experience bleeding during the course of anticoagulation therapy more often than non-cancer subjects [9], and administration of LMWH was shown to be associated with significantly higher risk of haemorrhagic complications compared to placebo or lack of VTE prevention [12].

Published meta-analyses [8, 9, 12, 13] confirmed that the efficacy of LMWH in the prevention of VTE is higher than the efficacy of vitamin $K$ antagonists (VKA), inhibitors of factor Xa (rivaroxaban, apixaban, edoxaban) and thrombin inhibitors (dabigatran), whereas the safety profiles of these therapies seem to be similar. Since available evidence does not suggest that the safety profile of novel anticoagulants is better than that of $\mathrm{LMWH}$, the latter remain the treatment of choice in cancer patients. Routine treatment of VTE includes sequential administration of LMWH followed by VKA or new anticoagulant drugs. The only exceptions pertain to cancer patients, pregnant women, individuals at increased risk of bleeding, and those in whom prothrombin time or INR cannot be controlled due to various reasons; in all these groups, chronic administration of $L M W H$ is recommended $[10,11,14]$. A recently published meta-analysis [15] demonstrated that LMWH is more efficient than VKA in the secondary prevention of VTE. The potential risk of heparin-induced thrombocytopaenia (HIT) does not negatively affect the safety profile of LMWH $[16,17]$. Moreover, administration of LMWH in the general population was shown to be associated with lower incidence of haemorrhagic complications than the use of other anticoagulant therapies [17]. Castellucci et al. [17] conducted a meta-analysis of 45 studies comparing the efficacy and safety of various anti-VTE protocols (LMWH-VKA, unfractionated heparin-VKA, fondaparinux-VKA, LMWH-dabigatran, LMWH-edoxaban, apixaban, rivaroxaban, or LMWH in monotherapy). They did not find significant differences in the safety profiles of the analysed treatments, aside from a slightly higher risk of recurrent VTE after administration of unfractionated heparin with VKA. The same study demonstrated that the use of in- hibitors of factor Xa (apixaban, rivaroxaban) or LMWH in monotherapy is associated with lower risk of haemorrhagic complications [17]. These findings were further confirmed by Alikhan et al. [18], who showed that LMWH is more efficient than unfractionated heparin in the prevention of deep vein thrombosis, and their use is associated with lower risk of haemorrhagic complications. However, none of the differences turned out to be statistically significant [18], and therefore, available evidence is insufficient to draw any conclusion regarding the superiority of $\mathrm{LMWH}$ in VTE prevention.

In conclusion, the results of this multicentre observational study imply that administration of LMWH is a safe form of pharmacological prophylaxis for VTE in surgically-treated cancer patients.

The authors would like to express their gratitude to Dr. Szymon Bruzewicz (SciencePro) for his assistance in writing this manuscript.

The study was sponsored by Sanofi-Aventis Sp. z o.0. (Poland).

\section{References}

1. Lethen H, Flachskampf FA, Schneider R, et al. Frequency of deep vein thrombosis in patients with patent foramen ovale and ischemic stroke or transient ischemic attack. Am J Cardiol 1997; 80: 1066-9.

2. ec.europa.eu/eurostat/data/database.

3. Sandler DA, Martin JF. Autopsy proven pulmonary embolism in hospital patients: are we detecting enough deep vein thrombosis? J R Soc Med 1989; 82: 203-5.

4. Stein PD, Henry JW. Prevalence of acute pulmonary embolism among patients in a general hospital and at autopsy. Chest 1995; 108: $978-81$.

5. Stein PD, Henry JW. Clinical characteristics of patients with acute pulmonary embolism stratified according to their presenting syndromes. Chest 1997; 112: 974-9.

6. Ageno W, Squizzato A, Garcia D, Imberti D. Epidemiology and risk factors of venous thromboembolism. Semin Thromb Hemost 2006; 32: 651-8.

7. Agnelli G, Verso M. Management of venous thromboembolism in patients with cancer. J Thromb Haemost 2011; 9 Suppl 1: 316-24.

8. Cohen AT, Agnelli G, Anderson FA. Venous thromboembolism (VTE) in Europe. The number of VTE events and associated morbidity and mortality. Thromb Haemost 2007; 98: 756-64.

9. Di Nisio M, Porreca E, Otten HM, Rutjes AW. Primary prophylaxis for venous thromboembolism in ambulatory cancer patients receiving chemotherapy. Cochrane Database Syst Rev 2014; 29 : CD008500.

10. Carrier M, Cameron C, Delluc A, Castellucci L, Khorana AA, Lee AY. Efficacy and safety of anticoagulant therapy for the treatment of acute cancer-associated thrombosis: a systematic review and meta-analysis. Thromb Res 2014; 134: 1214-9.

11. Pruthi RK. Review of the American College of Chest Physicians 2012 Guidelines for Anticoagulation Therapy and Prevention of Thrombosis. Semin Hematol 2013; 50: 251-8.

12. Zawilska K, Jaeschke R, Tomkowski W, et al. Polskie wytyczne profilaktyki i leczenia żylnej choroby zakrzepowo-zatorowej. Pol Arch Med Wewn 2009; 119 (suppl 1): 1-69.

13. Che DH, Cao JY, Shang LH, Man YC, Yu Y. The efficacy and safety of low-molecular-weight heparin use for cancer treatment: a meta-analysis. Eur J Intern Med 2013; 24: 433-9.

14. Akl EA, Barba M, Rohilla S, Terrenato I, Sperati F, Muti P, Schünemann HJ. Low-molecular-weight heparins are superior to vitamin $\mathrm{K}$ antagonists for the long term treatment of venous 
thromboembolism in patients with cancer: a cochrane systematic review. J Exp Clin Cancer Res 2008; 27: 1756-66.

15. AkI EA, Kahale L, Neumann I, Barba M, Sperati F, Terrenato Muti P Schünemann H. Anticoagulation for the initial treatment of venous thromboembolism in patients with cancer. Cochrane Database Syst Rev 2014; 19: CD006649.

16. Bochenek T, Nizankowski R. The treatment of venous thromboembolism with low-molecular-weight heparins. A meta-analysis. Thromb Haemost 2012; 107: 699-716.

17. Castellucci LA, Cameron C, Le Gal G, et al. Clinical and safety outcomes associated with treatment of acute venous thromboembolism: a systematic review and meta-analysis. JAMA 2014; 312 1122-35.

18. Alikhan R, Bedenis R, Cohen AT. Heparin for the prevention of venous thromboembolism in acutely ill medical patients (excluding stroke and myocardial infarction). Cochrane Database Syst Rev 2014; 7: CD003747.

\section{Address for correspondence}

\section{Tomasz Olesiński}

Department of Oncological Gastroenterology

Maria Sklodowska-Curie Memorial Cancer Center

and Institute of Oncology

Roentgena 5

02-781 Warsaw, Poland

e-mail: tolesinski@coi.waw.pl

Submitted: 22.03 .2017

Accepted: $\quad 25.03 .2017$ 